\title{
Erratum to: Rice GDP-mannose pyrophosphorylase OsVTC1-1 and OsVTC1-3 play different roles in ascorbic acid synthesis
}

\author{
Hua Qin ${ }^{1,2} \cdot$ Zaian Deng $^{1,3}$ - Chuanyu Zhang ${ }^{1,3} \cdot$ Yayun Wang ${ }^{1,2} \cdot$ Juan Wang $^{1,2}$. \\ Hai Liu ${ }^{4}$. Zhili Zhang ${ }^{3} \cdot$ Rongfeng Huang ${ }^{1,2} \cdot$ Zhijin Zhang $^{1,2}$
}

Published online: 16 February 2016

(C) Springer Science+Business Media Dordrecht 2016

\section{Erratum to: Plant Mol Biol (2016) 90:317-327 DOI 10.1007/s11103-015-0420-0}

Due to an unfortunate turn of events, the Acknowledgments section was omitted in the original publication, causing the funding agencies not to be properly acknowledged. The correct information is published here and should be treated as definitive.

\begin{abstract}
Acknowledgments This work was supported by the National Research Program of China (2013CB127003), the Major Special Foundation of Transgenic Plants in China (2014ZX08001-002 and 2014ZX08009-15B), and the National Natural Science Foundation of China (Grant No. 31070270).
\end{abstract}

The online version of the original article can be found under doi:10.1007/s11103-015-0420-0.

Rongfeng Huang

rfhuang@caas.cn

$\triangle$ Zhijin Zhang

zhangzhijin@caas.cn

1 Biotechnology Research Institute, Chinese Academy of Agricultural Sciences, Beijing 100081, China

2 National Key Facility of Crop Gene Resources and Genetic Improvement, Beijing 100081, China

3 Hainan Academy of Agricultural Sciences, Haikou 571000, China

4 Department of Biology, University of Virginia, Charlottesville, VA 22903, USA 